\title{
Hanging by a thread: choosing the right thread for the right patient
}

Keywords: surgery, polydioxanone, polylactic acid, orthopaedic, polycaprolactone, hyaluronic acid

Abbreviations: PDO, polydioxanone; PLA, polylactic acid; PCA, polycaprolactone

\section{Mini review}

Ageing causes loss of facial fat, especially around the cheeks, the eye area, the jowls and the neck. Accompanying this is skin ageing where the elastic fibers in the skin become thinner resulting in loss of facial elasticity? The two processes result in a longer face an

wrinkles due to the "facial scaffolding"not being able to provide as much support. Thread lifts or suture lifts involve the use of threads or sutures made from materials used in surgery to close wounds. When threads are placed under the skin they can tighten and lift loose or sagging areas in various parts of the face and body to help reduce the effects of gravity and ageing, or they can be used to rejuvenate the face. ${ }^{1}$

There are three main types of threads currently available; polydioxanone (PDO), polylactic acid (PLA) and polycaprolactone (PCA). PDO threads have been around the longest and are made of a synthetic biodegradable polymer that has been used in surgery for many years. PDO threads are absorbed into the body over 6 months by hydrolysis and work by triggering fibroblasts to produce more collagen in a targeted area. There are three main types of PDO threads used; mono, $\operatorname{cog}$ and screw threads. Mono threads are smooth without barbs and are anchored to a point on the face or the scalp. They mainly tighten the skin and provide a small amount of lift. $^{2}$ Cog threads have barbs which hook onto the skin to provide support and lift the sagging tissue. Screw threads have one or two intertwined threads around the needle and provide good volume restoration to sunken areas of the skin. The production of collagen around the threads and their barbs helps to restore volume and improves the skin texture and elasticity resulting in a natural aesthetics outcome.

After PDO threads, PLA threads were developed. They are made from a biocompatible polymer derived from lactic acid that has been used in many applications such as orthopaedic pins and sutures. PLA threads are resorbable and regenerate collagen over a longer time than PDO threads. PLA threads use cones to hook to the tissue and increase the volume of saggy areas therefore helping to restore shape to the facial area as well as providing a lift.

PCA threads are the newest threads and are bio-absorbable, monofilament suspension threads of synthetic origin (caprolactone). ${ }^{3}$ They work by regenerating collagen over a longer time than PDO and PLA threads. They leave behind a collagen structure that provides support for the skin, tightens the skin and prevents it from sagging. Due to the fibrotic reaction caused by the threads, the lifting and stretching action will continue even after the threads have been resorbed. The process of thread breakdown produces molecules of small molecular weight which subsequently induce the production of
Volume I Issue 4 - 2017

\author{
Vincent Wong, Rafiq N, Kalyan R, Hsenriksen \\ A, Funner R \\ Derma Clinic, London
}

Correspondence: Vincent Wong, Derma Clinic, London, Tel 0207299 0380,Email info@drvincentwong.com

Received: May 15, 2017| Published: December 05, 2017

collagen and Hyaluronic acid by the skin. The resulting skin is more moisturized, revitalized and firm with a long lasting result.

Before deciding what type of thread to use we must consider the treatment indications and what we are trying to achieve; facial lift or facial rejuvenation. ${ }^{4}$ If a patient wanted tissue lifting PDO monofilament threads would be unsuitable as they are placed superficially, are completely smooth and without barbs. While they produce a regenerative and firming effect that visibly improves skin quality they do not provide an effective amount of tissue lifting. ${ }^{5}$ To achieve a powerful lift with improvement in facial tightening and rejuvenation, barbed threads must be used. The barbs along the threads act as cogs to clasp the skin creating tension in the thread which lifts and suspends the facial area. Collagen is formed around the threads and their barbs resulting in an increased effect. ${ }^{6,7}$ Consider now a patient, who requires a facial lift, there are further factors which ensure efficacy and longevity of the results (barb length, angle, spatial distribution and direction of barbs or cones, resorption time and the collagen-stimulating ability of the thread).

The barbs must have a length that enables them to hook onto the skin tissue and maintain the lift required. If they are too long then they become too flexible and incapable of lifting the facial tissue. On the other hand, if the barbs are too short they will not be able to hook onto the facial tissue in the first instance. Furthermore, we need to consider how densely the barbs are placed along the thread length. Threads with a low barb density will not be able to lift the same amount of tissue as threads with a high barb density and therefore will not result in the desired facial lift. In addition, low barb density threads will be less effective at lifting larger volumes of tissue or heavier tissue and restrict the practitioner to working only with small amounts of soft tissue. Of course, a thread must have smooth areas that are barb-free to ensure there is adequate anchoring and to avoid any puckering of the skin.

The angles of the barbs also have a place in how much hold is achieved. If the angle is too small then the lift will be weak, conversely, if the angle is too big the barb may dig into the thread causing it to break. The manufacturing process for some threads can result in the barbs actually digging into the thread, reducing its thickness or gauge in areas. Ideally, the length of the base of the barb should be equal to the thickness of the thread. The spatial distribution of the barbs 
along the thread will also vary and the more contact there is between the barbs and the facial tissue, the better the hold. Some threads have barbs at different angles in order to achieve a $360^{\circ}$ lift; some have cones to maximize tissue contact, while others have all the barbs lined up in one line only.

Threads can come as either mono-directional or bi-directional. To achieve effective lifting of facial tissue the bi-directional threads are preferred as they provide immediate anchoring to the tissue and the thread cannot move either way due to the two-way direction of the barbs. Some mono-directional threads are anchored on both ends at fixed points to enhance stability. ${ }^{8}$ The final consideration to make is how long the thread will last in the tissue. PDO threads will stay in the tissue for around 6 months, PLA threads around 12 months, and PCA threads will stay in the tissue for 12-15 months. The longer the thread lasts the more collagen is stimulated and therefore the result is much better and longer lasting. PDO and PLA threads cause fibrosis in the surrounding area and create type 1 collagen. PCA threads stimulate the production of type 1 and 3 collagen which helps to improve the condition of the skin giving a youthful appearance.

How do we decide which thread to use for which patient? Ultimately we have to look at the age of the patient, the treatment area and what we are trying to achieve PDO threads are better at repositioning and revitalizing tissue but not for providing lift so would be suited more to younger patients. PLA threads provide some lifting, but again would be suitable for patients who only require a small amount of tissue lift. PCA threads provide more lift and are more suitable for patients who require a small to moderate amount of lift. Other factors that have an effect on the desired results are the technique used to insert the threads and the positioning of the threads (Figure 1). ${ }^{9}$

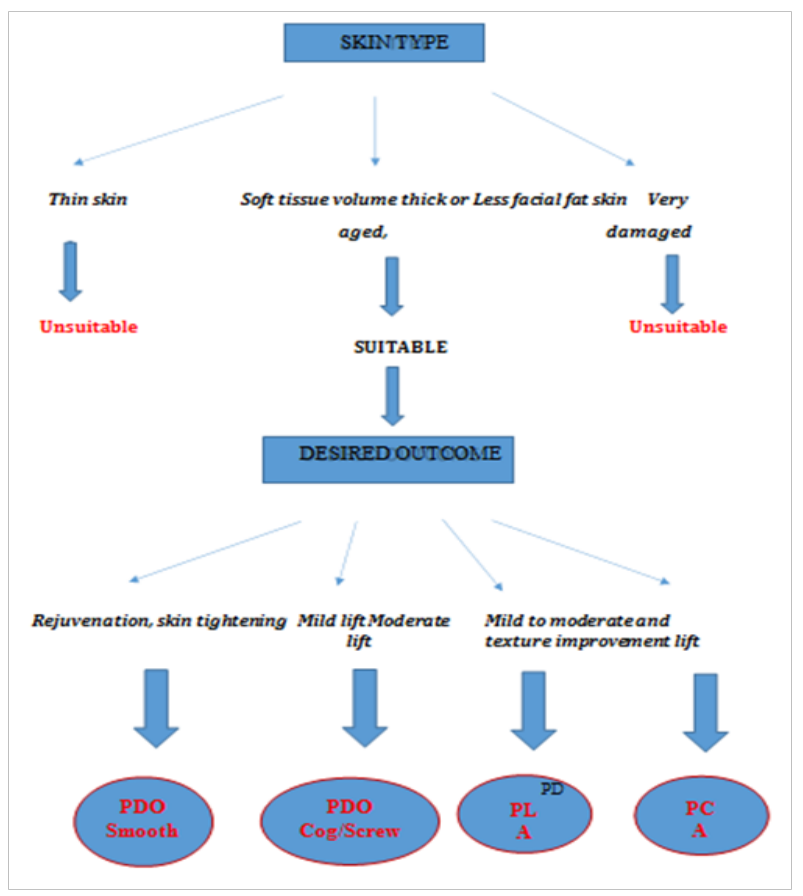

Figure 1 Decision tree to select the right thread for the right patient.
In summary, each thread type has a place and selecting the right thread for the right patient is vital to achieve the desired outcome as well as managing the patient's expectations. Good skin is essential as response to the treatment relies on the threads to tighten over the lifted area. Patients with thin skin may have more chances of sutures showing, rippling effect and bruising. ${ }^{10}$ Threads lifts are not suitable for patients with excessively saggy skin. Threads may not be suitable if the skin is very aged, thick or damaged, but still it is important to remember that thread lift, especially PCA, represents an option for those who cannot tolerate surgical lifting or narcosis. Patients with good soft tissue volume, less facial fat and a small amount of skin to be lifted will benefit the most from thread lifts. For patients who desire a lifting and/or revitalization effect, thread lifting is a minimally invasive technique which is well-tolerated. The procedure is quick and mostly pain free, although the outcome and final results are dependent on the qualifications discussed above. There will be patients for whom surgical lifting will be a more suitable option and most importantly, we must remember that thread lifts are not designed to replace surgical lifting (Table 1).

Table 1 Summarizing the different threads

\begin{tabular}{llllll}
\hline $\begin{array}{l}\text { Thread } \\
\text { type }\end{array}$ & Rejuvenation & $\begin{array}{l}\text { Mild } \\
\text { lift }\end{array}$ & $\begin{array}{l}\text { Mild to } \\
\text { moderate } \\
\text { lift }\end{array}$ & $\begin{array}{l}\text { Moderate } \\
\text { lift }\end{array}$ & Longevity \\
\hline PDO & $\mathrm{X}$ & & & & 6 months \\
Smooth & & $\mathrm{X}$ & & & 6 months \\
PDO & $\mathrm{X}$ & & & & \\
Cog/ & & & $\mathrm{X}$ & & 12 months \\
Screw & & & & $\mathrm{X}$ & $\begin{array}{l}12-15 \\
\text { months }\end{array}$ \\
PLA & $\mathrm{X}$ & & & \\
PCA & $\mathrm{X}$ & & &
\end{tabular}

\section{Acknowledgements}

None.

\section{Conflict of interest}

The author declares no conflict of interest.

\section{References}

1. Khazanchi R, Aggarwal A, Johar M. Anatomy of ageing face. Indian $J$ Plast Surg. 2007;40(2):223-229.

2. Shimizu Y, Terase K. Thread lift with absorbable monofilament threads. $J$ Japan Soc Aesthetic Plast Surg. 2013;35(2):1-12.

3. Savoia A, Accardo C, Vannini F, et al. Outcomes in thread lift for facial rejuvenation: a study performed with happy lift ${ }^{\mathrm{TM}}$ revitalizing. Dermatology and Therapy. 2014;4(1):103-114

4. Tonks S. Understanding thread lifting. Aesthetics. 2015.

5. Dancey E. New generation absorbable threads. Aesthetics. 2014.

6. Wu WT. Barbed sutures in facial rejuvenation. Aesthet Surg J. 2004;24(6):582-587.

7. De Lorenzi C. Barbed sutures: Rationale and technique. Aesthet Surg J. 2006;26(2):223-229. 
8. Kalra R. Use of barbed threads in facial rejuvenation. Indian Journal of Plastic Surgery. 2008;41(Suppl):S93-100.

10. Paul MD. Complications of barbed sutures. Aesthet Plast Surg. 2008;32(1):149.

9. Mian I. PDO Threadlifting. Aesthetics. 2017. 